\title{
Top-down knowledge modulates onset capture in a feedforward manner
}

\author{
Stefanie I. Becker ${ }^{1}$ - Amanda J. Lewis ${ }^{1}$ - Jenna E. Axtens ${ }^{1}$
}

Published online: 17 August 2016

(C) Psychonomic Society, Inc. 2016

\begin{abstract}
How do we select behaviourally important information from cluttered visual environments? Previous research has shown that both top-down, goal-driven factors and bottom-up, stimulus-driven factors determine which stimuli are selected. However, it is still debated when top-down processes modulate visual selection. According to a feedforward account, top-down processes modulate visual processing even before the appearance of any stimuli, whereas others claim that top-down processes modulate visual selection only at a late stage, via feedback processing. In line with such a dual stage account, some studies found that eye movements to an irrelevant onset distractor are not modulated by its similarity to the target stimulus, especially when eye movements are launched early (within 150-ms post stimulus onset). However, in these studies the target transiently changed colour due to a colour after-effect that occurred during premasking, and the time course analyses were incomplete. The present study tested the feedforward account against the dual stage account in two eye tracking experiments, with and without colour after-effects (Exp. 1), as well when the target colour varied randomly and observers were informed of the target colour with a word cue (Exp. 2). The results showed that top-down processes modulated the earliest eye movements to the onset distractors ( $<150$-ms latencies), without incurring any costs for selection of target matching distractors. These results unambiguously support a feedforward account of topdown modulation.
\end{abstract}

Stefanie I. Becker

s.becker@psy.uq.edu.au

1 School of Psychology, The University of Queensland, Brisbane, Australia
Keywords Attentional capture - Eye movements and visual attention · Visual search $\cdot$ Cognitive control and automaticity

It is well known that visual selection is determined by both top-down, goal-driven factors and bottom-up, stimulus-driven factors (Wolfe, 1994). Visually salient objects that pop out from the surround can automatically attract attention and the gaze in a purely stimulus-driven manner, independently of our goals and intentions (Theeuwes, 1992, 1994; Theeuwes, Atchley \& Kramer, 2000). On the other hand, knowledge about the features of the relevant object (target) can limit selection to stimuli that are similar to goal-relevant objects in a top-down controlled manner (Folk, Remington \& Johnston, 1992). Most theories of visual attention assume that bottomup and top-down factors interact in visual selection (Wolfe, 1994). Still, a hotly debated question is whether top-down processing can modulate visual processing during feedforward processing or whether it affects selection only at a later stage, via feedback connections (Ansorge, Horstmann \& Scharlau, 2010; Theeuwes, 2013).

One widespread view is that top-down processes can modulate sensory processing and visual selection in a feedforward manner. According to this view, the intention to select a specific stimulus increases our sensitivity to the sought-after feature even before the appearance of any stimuli, so that corresponding sensory inputs are already modulated during feedforward processing (Ansorge et al., 2010; Folk et al., 1992; Wolfe, 1994). By contrast, according to the dual stage account (Theeuwes et al., 2000), visual selection is initially, at an early stage, completely determined by bottom-up saliency signals. Top-down knowledge modulates visual selection only at a later stage, via feedback processing.

The feedforward view often has been tested against the dual stage account by comparing responses to suddenly 
appearing objects (onsets; Ludwig \& Gilchrist, 2002; Mulckhuyse, van Zoest \& Theeuwes, 2008; Yantis \& Jonides, 1984). An onset or the sudden appearance of a stimulus is thought to generate an early, strong bottom-up signal that can automatically attract attention to its location (Yantis \& Jonides, 1984). Yet, target-similar onset distractors (e.g., onsets with the same colour as the target) typically attract the gaze more strongly (e.g., on $49 \%$ of all trials) than targetdissimilar onset distractors (e.g., green onsets; $7 \%$ : see Ludwig \& Gilchrist, 2002, Experiment 1). These results suggest that capture by irrelevant onsets is modulated by topdown processes (Becker \& Lewis, 2015; Geyer, Mueller \& Krummenacher, 2008; Ludwig \& Gilchrist, 2002; Moher et al., 2011, Sayim et al., 2010; Wu \& Remington, 2003). Critically, however, Mulckhuyse and colleagues (2008) compared the time-course of saccades to target-similar and dissimilar onsets with a cumulative latency distribution analysis, and found that saccades to the target-similar onset did not start earlier, as one would expect on a feedforward account (where target-similar items should enjoy a selection advantage). Instead, target-similar onsets were selected with a small delay that was nonsignificant for the earliest saccades and increased with the latencies of saccades. Correspondingly, Mulckhuyse et al. (2008) concluded that the earliest eye movements were driven to the onsets in virtue of their sudden appearance, and that top-down processes modulated visual selection only at a later stage, via feedback connections (see also Van Zoest, Donk, \& Theeuwes, 2004).

However, in the cumulative distribution analysis, only the latencies of the 20-100\% earliest saccades to either onset were assessed (in steps of $20 \%$ ), without considering the possibility that a top-down bias could be reflected in a higher number of saccades to the target-similar onset. In fact, in Mulckhuyse et al.'s study (2008; Exp. 1), the target-similar onset was selected on $26 \%$ of all trials (i.e., on 100 trials), whereas the dissimilar onset was selected on only $8 \%$ of all trials (i.e., on 31 trials). Thus, within the $20 \%$ fastest eye movements, 20 were directed to the similar distractor and only 6 were directed to the dissimilar onset. More frequent selection of the targetsimilar onset with the earliest eye movements could arguably reflect an effect of top-down tuning of attention and could be in line with a feedforward account of top-down control; yet, these differences were not assessed in the cumulative latency distribution analysis.

If early eye movements to an onset distractor were solely driven by bottom-up processes, both the number of eye movements and their latency to target-similar and target-dissimilar distractor should be the same, in contrast to the finding that typically, more eye movements are made to a target-similar onset. To date, only a single study has reported equal selection rates for target-similar and dissimilar onsets. Specifically, a study by Born, Kerzel, and Theeuwes (2011) induced very fast eye movements to onset distractors (with average latencies between $140 \mathrm{~ms}$ and $165 \mathrm{~ms}$ ) and found that targetsimilar and target-dissimilar onsets were selected to the same extent ( $\sim 45 \%$ of all trials). This result indeed seems to contradict the feedforward view and constitutes the perhaps best available evidence for the dual stage account - that early saccades are not modulated by top-down processes.

However, a problematic aspect of these results is that the findings of Born et al. (2011) could have been due to a colour after-effect. Of note, the premasks were all red, and the target was defined by a colour change to grey (Mulckhuyse et al., 2008). Presenting a grey stimulus in the same position as a coloured premask stimulus can produce a colour after-effect, so that a grey target stimulus initially appears to be greenish blue before it fades to grey (due to chromatic adaptation of the "red" cones; see Theeuwes and Lucasson, 1993 and Theeuwes, 2004, footnote 1). It is possible that this subjective impression of a transient colour change impaired top-down tuning to the target, either because observers tuned attention to multiple different colours or any salient colour difference (e.g., singleton search mode; Bacon \& Egeth, 1994; Harris, Becker \& Remington, 2015), or by impairing filtering of taskirrelevant distractors (Folk \& Remington, 1998). Of note, in some studies, the target-dissimilar onset distractor could also be blue or green (Born et al., 2011), and thus was potentially similar to the perceived target. Hence, in these studies, capture by the onset distractor may not have been due to bottom-up, saliency-driven processes, but to top-down processes.

The purpose of the first experiment was to examine critically whether previous results suggesting equal selection rates of similar and dissimilar distractors could be due to top-down tuning to an illusory target colour. In a second experiment, we presented the target without a colour after-effect and compared a cumulative and noncumulative latency distribution analysis to assess whether top-down knowledge affect the proportion and/or latencies of the earliest saccades to an onset distractor.

\section{Experiment 1}

In Experiment 1, we examined the effects of the colour aftereffect on selection, by presenting the target with or without a colour after-effect in two blocked conditions. In our laboratory, presenting a grey target against the background of red premasks evoked the impression that the target was initially blue and then faded into grey. To assess whether presenting the target with this subjective colour change impairs distractor filtering or leads observers to adopt a singleton detection mode, we tested four different onset distractors: a targetsimilar (grey) onset, an onset that was similar to the induced colour of the target (blue onset), a target-dissimilar green onset, and an onset that was similar to the other nontargets and premasks (red onset). Target and distractor selection in this red 
premask condition was then compared to a blocked condition in which the target stimulus was presented without a colour after-effect: In the grey premask condition, the premasks were initially all grey and the target was defined by a colour change to red, which did not produce an after-effect (as chromatic adaptation is equal across all cones; Theeuwes and Lucassen, 1993).

If observers bias attention to all singletons in the red premask condition (or all colour singletons; Harris et al., 2015), then all onset distractors (or all differently coloured onset distractors) should attract the gaze more strongly, compared with the grey premask condition. On the other hand, if observers bias attention narrowly to the two possible target colours, then only the two target-similar (grey and blue) distractors should strongly attract the gaze in the red premask condition, and selection rates of the target-dissimilar distractors (green and premask coloured) should not differ across the red and grey premask condition.

\section{Method}

Participants Twenty-four naïve observers from The University of Queensland participated in the experiment in exchange for a $\$ 10$ payment. Four of the observers were male (20 females), and their mean age was 22.58 years (range: 20 33 years; standard deviation $[S D]: 3.20$ ).

Apparatus A BenQ 19" LCD colour monitor (resolution: $1,280 \times 1,024$ pixels; refresh rate: $75 \mathrm{~Hz}$ ) and a Dell Optiplex 745 computer running Presentation software (Neurobehavioral Systems) were used to control the experiment and display the stimuli. A video-based eye tracker (Eyelink 1000, SR Research, Ontario, Canada) recorded eye movements with a spatial resolution of $0.1^{\circ}$ and a temporal resolution of $500 \mathrm{~Hz}$.

Stimuli All stimuli were presented against a black background. The fixation display consisted of a white cross (size: $0.27^{\circ} \times 0.27^{\circ}$ ) presented at the centre of the screen. The premask display consisted of the fixation cross and six coloured disks (diameter: $1.38^{\circ}$ ) that were distributed evenly on the outlines of an imaginary circle with a diameter of $17.7^{\circ}$ (Fig. 1). On distractor present trials, the target display additionally included a red ( $\left.L u^{\prime} v^{\prime}: 17.9,0.325,0.547\right)$, blue ( $L u^{\prime} v^{\prime}$ : $17.4,0.149,0.356$ ), green ( $\left.L u^{\prime} v^{\prime}: 17.8, .090, .569\right)$ or greycoloured square $\left(17.9,0.148,0.513\right.$; size: $\left.1.38^{\circ} \times 1.38^{\circ}\right)$ positioned between two nontarget stimuli in a previously empty location (onset distractor).

Design The experiment comprised the $2 \times 5$ within-subjects variables of premask colour (red vs. grey; blocked) and distractor colour (target-similar, blue, green, premask- coloured onset distractor; varied within each block). The distractor colour was varied such that the target occurred without a distractor on $20 \%$ of trials and equally often with one of the four differently coloured distractors (red, grey, green, and blue) on the remaining trials (20\% each). The target and distractor locations were chosen randomly on each trial, with the limitation that target and distractor did not occupy directly adjacent positions. All observers completed two blocks of 400 trials in counterbalanced order, for a total of 800 trials per participant.

Procedure Before the experiment, participants were instructed to make a fast and precise eye movement to the target and to press a mouse button while they were still fixating on the target. Each trial started with the presentation of the fixation display $(500 \mathrm{~ms})$, followed by the premask display. The target display was only presented when participants had maintained fixation on the fixation cross (within an area of $1.36^{\circ}$ ), for at least $500 \mathrm{~ms}$, plus a random period between 1 and $200 \mathrm{~ms}$. The target display was presented until the observer's manual response. Immediately afterwards, a feedback display appeared that informed participants about their saccade latency, and that additionally contained the warning "TOO SLOW" if participants took more than $300 \mathrm{~ms}$ to make an eye movement.

\section{Results}

Data Eye movements were parsed into saccades, fixations, and blinks using the standard parser configuration of the Eyelink. The first eye movement on a trial was attributed to the nearest stimulus (target, distractor, or nontarget) when it had crossed more than $60 \%$ of the distance to a stimulus ( $>5.43^{\circ}$ from the centre).

Trials were excluded from all analyses when observers had failed to move their eyes outside the fixation region within $1,000 \mathrm{~ms}$ or when they made an anticipatory eye movement $(<50 \mathrm{~ms})$, which led to a loss of $5.48 \%$ of all data. The vast majority of first eye movements ( $>95 \%$ ) was directed either to the target or the distractor. In the absence of a distractor, $98.2 \%$ of first eye movements were directed to the target, and there were no differences between the red and grey premask conditions ( $98.3 \%$ and $98.2 \%$ respectively), $t<1$. However, the target was selected significantly earlier in the grey premask condition $(208 \mathrm{~ms})$ than in the red premask condition $(234 \mathrm{~ms})$ in the absence of any distractors, $t(23)=$ $6.0, p<0.001$. Delayed selection of the target in the red premask condition could reflect difficulties of detecting a target with a subjective colour change or indicate that the red target (among the grey premasks) was perceptually more salient than the grey target (among the red premasks). 


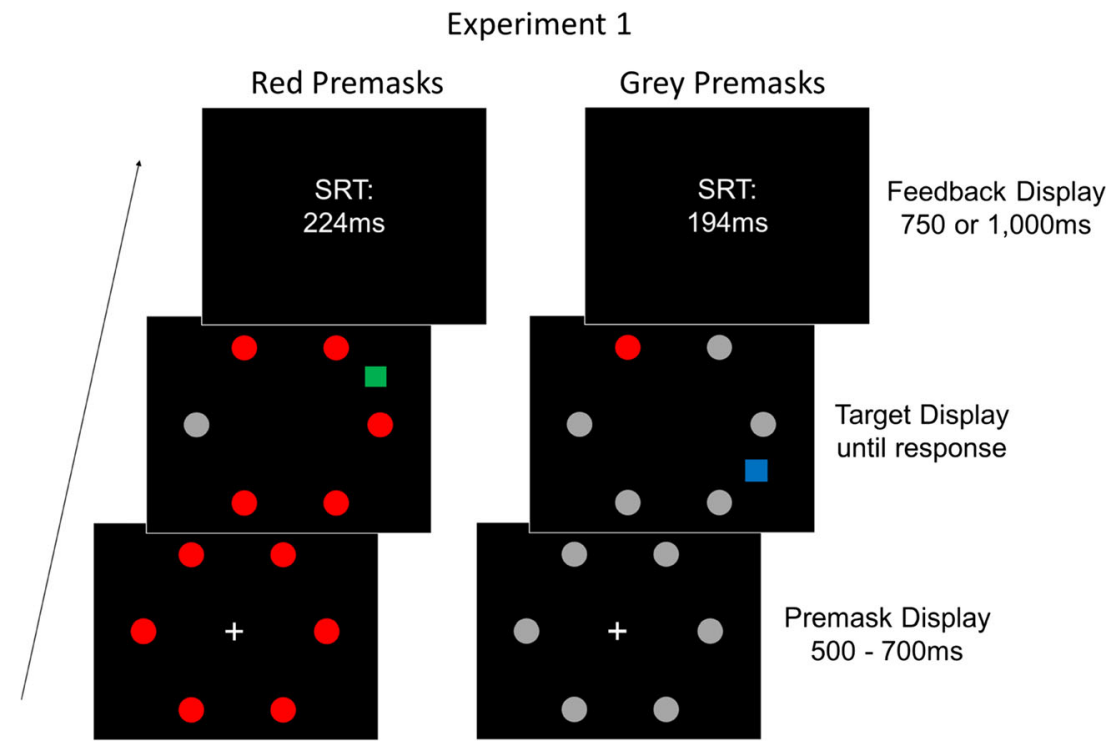

Fig. 1 Examples of trial types in the two conditions of Experiment 1. In the red premask condition, the target was defined by a colour change to grey, whereas in the grey premask condition, the target was a colour change to red. On $80 \%$ of all trials, an irrelevant onset distractor (square) was presented, which could be red, grey, green, or blue. Participants were instructed to make a fast and precise eye movement to

Proportion of first distractor fixations To compare the effects of different onset distractors across the conditions that included the colour after-effect (red premask) vs. not (grey premasks), a $2 \times 4$ repeated-measures ANOVA with the variables premask condition (red vs. grey premasks) and distractor colour (target-similar, blue, green, premaskcoloured) was computed over the proportion of first eye movements to the onset distractor. The results revealed significant main effects of the premask condition, $F(1,23)=145.8, p$ $<0.001, \eta^{2}=0.86$, the distractor colour, $F(3,69)=158.6, p<$ $0.001, \eta^{2}=0.87$, and a significant two-way interaction, $F(3,69)=39.3, p<0.001, \eta^{2}=0.63$. As shown in Fig. 2A, significantly more eye movements were made to all onset distractors in the red premask-condition, in which the target underwent the dynamic colour change than the grey premask condition (target-similar: $t(23)=6.6, p<0.001$, blue: $t(23)=$ $10.7, p<0.001$, green: $t(23)=10.4, p<0.001$, premaskcoloured: $t(23)=6.4, p<0.001)$.

This effect was especially pronounced for the blue distractor, in line with our impression that the target first appeared to be blue (before fading into grey). Specifically, in the red premask condition, the blue distractor was selected significantly more frequently than the green distractor, $t(23)=7.0, p$ $<0.001$, and the premask-coloured distractor, $t(23)=8.4, p<$ 0.001 . By contrast, in the grey premask condition, the blue distractor was not selected more frequently than the green distractor or the premask-coloured distractor, $t s<2.4$, ps $>$ 0.33 .

Yet, eye movements were still modulated by target similarity in both conditions, as the target-similar distractor attracted the target disk and to press a button when they were fixating on the target. A feedback display containing the words "Saccadic Response Time" and the time needed to make the eye movement (from the onset of the search display) was provided after each trial, followed by a blank display (for $250 \mathrm{~ms})$.

the gaze significantly more strongly than all other distractors, both in the red premask condition, all $t \mathrm{~S}>5.0, p \mathrm{~s}<0.001$, and in the grey premask condition, all $t \mathrm{~s}>9.2, p \mathrm{~s}<0.001$.

Cumulative Latency Distribution Analysis A cumulative latency distribution analysis as in Mulckhuyse et al. (2008) could only be conducted for the target-similar red and blue distractor in the red premask condition, as the other distractors were selected too infrequently. As shown in Fig. 2B, comparing the fastest $20 \%, 40 \%, 60 \%, 80 \%$, and $100 \%$ of saccades to the red vs. blue distractor revealed significant latency differences already in the fastest $20 \%$ of saccades, with significantly shorter latencies for the blue distractor than the targetsimilar distractor, $t(23)=4.3, p<0.001$. The latency difference steadily increased in later parts of the cumulative distribution, from initially $7 \mathrm{~ms}$ to $16 \mathrm{~ms}(40 \%: t(23)=6.0, p<$ $0.001 ; 60 \%: t(23)=6.5, p<0.001 ; 80 \%: t(23)=5.9, p<$ $0.001 ; 100 \%: t(23)=6.8, p<0.001$. In interpreting these results, it is important to note that the $20 \%$ fastest saccades consisted of 6.7 saccades to the blue distractor and 9.1 saccades to the target-similar distractor, and this difference was also significant, $t(23)=4.5, p<0.001$.

Noncumulative latency distribution analysis To assess whether the earliest saccades were biased to the targetsimilar onset, we conducted a second, noncumulative distribution analysis. For this analysis, trials with a target-similar, blue, green, or premask-similar distractor were ordered according to the latencies of the first saccade (to target or distractor) and sorted into five separate bins that contained 
A) Mean Distractor Fixations

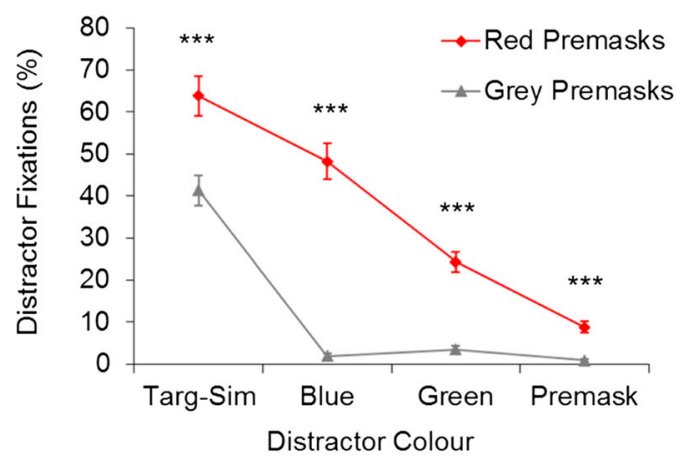

C)

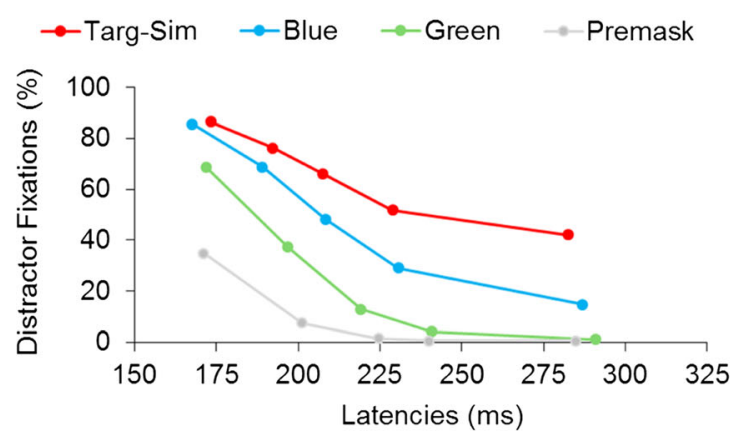

Fig. 2 A) Proportion of the first eye movements to each of the different onset distractors (Targ-Sim: target-similar onset, Premask: premask-coloured onset). Asterisks indicate significantly higher levels of capture in the red premask condition than in the grey premask condition. B) Distractor fixation latencies for the blue and target-similar, grey distractor in the red premask condition, depicted separately for the

the $0-20 \%$ fastest eye movements, $20-40 \%$ fastest eye movements, etc. Figures $2 \mathrm{C}$ and $\mathrm{D}$ depict the proportion of distractor fixations within each distractor condition, latency bin and premask condition. In this (noncumulative) distribution analysis, it is immediately clear that distractors were selected more frequently with the earliest saccades, whereas later saccades were more likely to be directed to the target. Second, with the exception of the blue distractor in the red premask condition, the target-similar distractor was always selected more frequently than the target-dissimilar distractor, across all bins and conditions, all $t \mathrm{~s}>3.5$, all $p \mathrm{~s} \leq 0.002$.

With regard to the saccadic latencies, this noncumulative distribution analysis is perhaps not very informative, as it includes eye movements to the target and the distractor (and a separate analysis was not possible as there were not enough fixations on the distractor in the majority of conditions). Still, in the red premask condition, the results also showed that the earliest eye movements were delayed in in the presence of a target-similar onset distractor compared with the blue distractor (bin 1: $168 \mathrm{~ms}$ vs. $173 \mathrm{~ms}$ ), $t(23)=6.2, p<0.001$, (bin 2: $188 \mathrm{~ms}$ vs. $192 \mathrm{~ms}$ ), $t(23)=2.7, p=0.012$ (bins 3-5: $\mathrm{t}<$ 1) but not compared to any of the other distractors. The targetsimilar distractor differed only from the green and premask-
B) Cumulative: Red Premasks
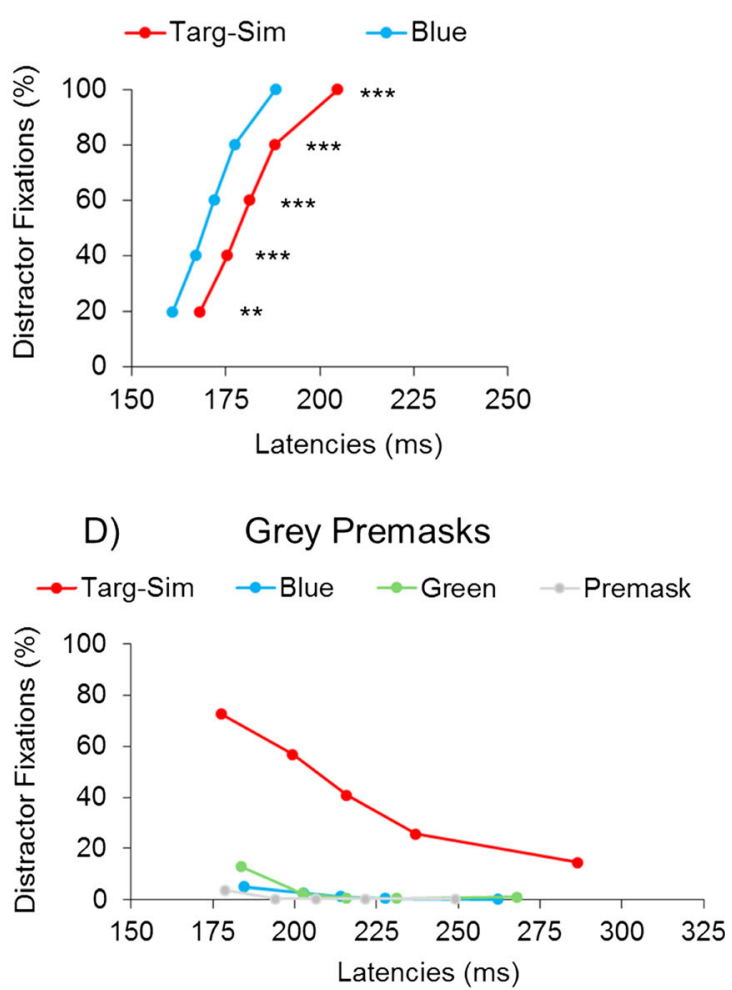

$20 \%-100 \%$ earliest eye movements (in steps of $20 \%$ ). C, D) Proportion of first fixations to the different onsets, depicted for the $20 \%$ earliest saccades, $20-40 \%$ earliest eye movements, etc., that went to either target or distractor $(100 \%)$. Error bars depict the Standard Error of the Mean (SEM) and may be smaller than the plotting symbol. $* * p<0.001$, $* * * p<0.00001$, two-sided $t$ test.

coloured distractors in later bins (bins 3 and 4 ) of the latency distributions (all $t \mathrm{~s}>2.3, p \mathrm{~s}<0.025$ ), indicating that a targetsimilar distractor interfered with later eye movements. The results were quite different for the grey premask condition, which showed that the earliest eye movements were significantly delayed in the presence of a blue distractor $(\mathrm{M}=$ $184 \mathrm{~ms})$ and a green distractor $(\mathrm{M}=184 \mathrm{~ms})$ compared with a target-similar distractor $(\mathrm{M}=178 \mathrm{~ms})$, both $t \mathrm{~s}=3.1, p \mathrm{~s}=$ 0.005 . Moreover, in line with the results above, the targetsimilar distractor delayed eye movements in the later part of the distribution (bins 4 and 5) compared with the blue distractor (bins 4 and 5: $t \mathrm{~s}>2.9, p \mathrm{~s} \leq 0.007$ ), green distractor (bin 5: $t(23)=3.9, p \leq 0.001)$, and premask-coloured distractor (bins 4 and 5: $t \mathrm{~s}>4.7, p \mathrm{~s}<0.001$ ).

\section{Discussion}

The results of Experiment 1 show clearly that an illusory colour change of the target can affect how attention is top-down tuned to the target. The appearance that the target was initially blue (before fading into grey) modulated visual selection in two ways: First, it selectively elevated selection of the blue 
onset distractor. Second, the illusory colour change led to generally elevated levels of selecting irrelevant onsets, regardless of their colour (Fig. 2A). Still, observers did not adopt a set for all salient items (singleton search mode; Bacon \& Egeth, 1994), which would have resulted in equally high selection rates for all differently coloured distractors (Folk \& Anderson, 2010; Harris et al., 2015). Instead, attention was apparently simultaneously tuned to the target colour (grey) and the illusory colour (blue). Tuning attention to two different colours (grey and blue) apparently impaired the observer's ability to effectively inhibit other irrelevant colours (e.g., green), for instance, because it depleted attentional resources (e.g., by inducing a higher cognitive load; Lavie et al., 2004). Alternatively, it is possible that distractor selection rates in the red premask condition were generally elevated, because the target was less salient (i.e., a grey target among red could be less conspicuous than a red target among grey).

Whereas distinguishing between these latter two possibilities would require further research, it seems to be clear that attention was actively biased to the subjective colour of the target (blue) as well as its veridical colour (grey), so that capture by the blue distractor cannot be attributed to its bottom-up saliency alone.

The results of the cumulative latency distribution analysis replicated the results of Mulckhuyse et al. (2008), in that selection of the target-similar (grey) distractor was already delayed in the $20 \%$ earliest eye movements and increased further with longer latency saccades (Mulckhuyse et al., 2008; Exp. 2). These results cannot be taken to support the dual stage account, because (1) the results suggest that attention was actively biased to blue, so that the comparison essentially involved two target-similar onset distractors, and (2) the results, especially from the grey premask condition, revealed a strong bias to select target-similar distractors over dissimilar ones, including with the earliest eye movements. These results appear to be more in line with a feedforward account of topdown control than the dual stage account.

The results of Experiment 1 do not yet provide conclusive evidence for a feedforward account, because the $20 \%$ fastest eye movements still had latencies above $150 \mathrm{~ms}$ (Fig. 2B-D). With this, the results are still consistent with the dual stage account, that top-down processes modulate eye movements only at a later stage. Experiment 2 was designed to probe more directly into early vs. late eye movements to clearly distinguish between the dual stage account and the feedforward view of top-down control.

\section{Experiment 2}

Experiment 2 differed from Experiment 1 in three important respects: First, only the grey premasks condition with the red target was used to avoid subjective colour changes of the target that could otherwise interfere with target selection or distractor filtering (thus rendering it difficult to distinguish between bottom-up and top-down processes). Second, only three differently coloured onset distractors (red, blue, and green) were used to increase the number of trials for the cumulative latency distribution analysis. Third, the target randomly had one of two possible colours (e.g., red or green), and observers were informed about the upcoming target colour before each trial with a word cue (e.g., RED or GREEN). These changes were introduced to allow assessing the effects of top-down knowledge on visual selection more definitely and, specifically, to exclude that selection of target-similar onset distractors can be attributed to automatic priming effects. Of note, previous studies on the feature priming effect (or priming of pop-out effect; Maljkovic \& Nakayama, 1994) have shown that selection of the target can prime attention and eye movements to select target-similar items on subsequent trials. Importantly, priming effects are not caused by top-down expectations, as the previous target's colour modulates attention even when observers are informed that the target will have a different colour on the next trial (Becker, 2007, 2008, 2010; but see Becker, Ansorge \& Horstmann, 2009). Thus, when the target always has the same colour across trials, it is possible that selection of a target-similar distractor is due to automatic priming effects, not top-down knowledge of the target colour.

In Experiment 2, we provided information about the upcoming target colour by a word cue and distinguished between automatic priming effects and genuine top-down processes by comparing the effects of the target-similar onset distractor (i.e., onset matching the word cue), the target-dissimilar onset (i.e., onset with a possible but not actual target colour), and unrelated onset (i.e., onset that never had the target colour) when the target vs. the distractor from the previous trial were repeated vs. not repeated. If selection of the target-similar onset is due to priming, then it should only be selected more frequently than the dissimilar onset when the target was repeated. In turn, if selection of the target-similar onset is due to top-down knowledge conveyed by the word cue, the targetsimilar onset should always be selected more frequently than the target-dissimilar and unrelated onsets (i.e., both on target repeat and nonrepeat trials). Moreover, if top-down tuning to the target modulates early visual processes (in a feedforward manner), the earliest eye movements should already be biased towards the target-similar onset distractor (i.e., eye movements with latencies below $150 \mathrm{~ms}$ ).

\section{Methods}

Participants Fourteen new observers comprising 2 males and 12 females (mean age: 21.57 years; range: $19-24$ years; $S D$ : 1.82) participated in the experiment in exchange of $\$ 10 /$ hour. 
Apparatus All stimuli were displayed on a 17" Samsung SyncMaster 957 CRT monitor with a resolution of $1280 \times$ 1024 pixels and a refresh rate of $85 \mathrm{~Hz}$, using an Intel Core 2 Quad CPU with $2.83 \mathrm{GHz}$ and $3.12 \mathrm{~GB}$ RAM. A standard USB keyboard and USB mouse were used as response devices.

Stimuli and Design These were the same as in the previous experiment, with the following exceptions: First, the premasks (diameter: $1.3^{\circ}$; distance from fixation: $8.1^{\circ}$ ) in Experiment 2 were always grey ( $L u^{\prime} v^{\prime}: 16.32, .205, .449$ ), and the target randomly had two of three possible colours (e.g., red or green, but not blue). Before each trial, a word cue indicated the colour of the upcoming target with $100 \%$ validity. On every trial, an onset distractor was presented (square shape, diameter: $1.2^{\circ}$ ) that could be either red (Lu'v': 15.87, 0.284, 0.508), green (Lu'v': 16.73, $0.158,0.548)$, or blue (Lu'v': 16.2, 0.189, 0.238). The onset distractor was referred to as the similar, dissimilar or unrelated distractor, depending on whether it had the same colour as the target, a possible (but noncued) target colour, or a colour that was never associated with the target. The colours of the similar, dissimilar, and unrelated distractors varied across participants, and participants completed 720 trials during the experiment.

Procedure The procedure was identical to Experiment 1, except that before each premask display, a word cue was presented (RED, GREEN, or BLUE; the target could have two colours that varied across participants). Participants were instructed to press the spacebar with the left hand when they had read the word. Immediately afterwards, a central white fixation cross was presented for $500 \mathrm{~ms}$, followed by the premask display, which consisted of the word cue surrounded by the six grey premasks. The search display was presented only when the gaze had been in the centre for at least $700 \mathrm{~ms}$, plus a randomly chosen duration between 1 and $200 \mathrm{~ms}$ (Fig. 3A).

\section{Results}

Data Excluding trials in which the first fixation could not be assigned to a target or nontarget location resulted in a loss of $1.5 \%$ of all trials. Excluding trials in which the eyes had moved outside the fixation region before $50 \mathrm{~ms}$ or after $1000 \mathrm{~ms}$ led to an additional loss of $0.2 \%$ of all data. As in Experiment 1, the majority of first eye movements was directed to the target or the onset distractor ( $>91 \%$ of first eye movements across all conditions).

First eye movements to the distractor A three-way, repeated-measures ANOVA comparing selection rates across the three onset distractor conditions (similar, dissimilar, unrelated) showed a significant main effect of the distractor condition, $F(2,26)=42.2, p<0.001, \eta^{2}=$ 0.77 . The similar distractor was selected significantly more frequently than the dissimilar distractor, $t(13)=$ 5.8, $p<0.001$, and the unrelated distractor, $t(13)=7.5$, $p<0.001$. The dissimilar distractor was also selected significantly more frequently than the unrelated distractor, $t(13)=3.5, p=0.004$, which had a colour that was never the target colour.

Priming Effects To assess whether higher selection rates of the similar distractor may have been mediated by priming (i.e., target or distractor repetition), the proportion of first eye movements to each distractor was subjected to a $3 \times 2 \times 2$ ANOVA comprising the variables distractor type (similar, dissimilar, unrelated), target repetition (target repeated vs. not repeated) and distractor repetition (onset repeated vs. not repeated). The results showed significant main effects of distractor type, $F(2,26)=40.0, p<0.001, \eta^{2}=0.76$, and target repetition, $F(1,13)=8.8, p=0.011, \eta^{2}=0.41$, as well as a significant interaction between the two variables, $F(2,26)=$ 4.2, $p=0.035, \eta^{2}=0.25$ (all other $p \mathrm{~s}>0.17$ ).

Target repetition modulated capture only by the dissimilar distractor, $t(13)=3.2, p=0.007$, but not the similar or unrelated distractor, $t \mathrm{~s}<1.5, p \mathrm{~s}>0.18$ (Fig. 3B). For the dissimilar distractor, selection rates were higher on target switch trials, in which the distractor (but not the current target) matched the previous target. Still, across all repetition conditions, the target-similar distractor was selected significantly more frequently than the dissimilar distractor, $t \mathrm{~s}>3.6, p s \leq$ 0.003 , and the unrelated distractor, $t \mathrm{~s}>6.1, p \mathrm{~s}<0.001$. With this, higher selection rates of the target-similar distractor cannot be attributed to priming effects, but are due to top-down tuning to the target colour (by the word cue).

Cumulative Latency Distribution Analysis Analysing the data with the same cumulative latency distribution analysis as in Mulckhuyse et al. (2008) required excluding two subjects who had selected one of the distractors on less than five occasions. The results of the remaining 12 subjects showed that eye movements to the target-similar distractor were significantly delayed compared with the dissimilar and unrelated distractor, across all bins (all $t \mathrm{~s}$ $>3.7, p \mathrm{~s}<0.003$ ), and the effect increased in magnitude from the earliest bin (7-ms difference) to the last bin (13ms difference; Fig. 3C). The dissimilar distractor also was selected significantly later than the unrelated distractor, across all bins, all $t \mathrm{~s}>2.6, p \mathrm{~s}<0.024$, and this effect, 


\section{A) Experiment 2}

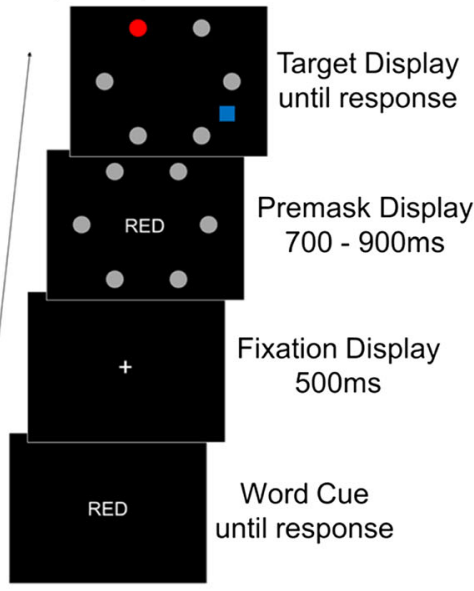

C) Cumulative Distribution

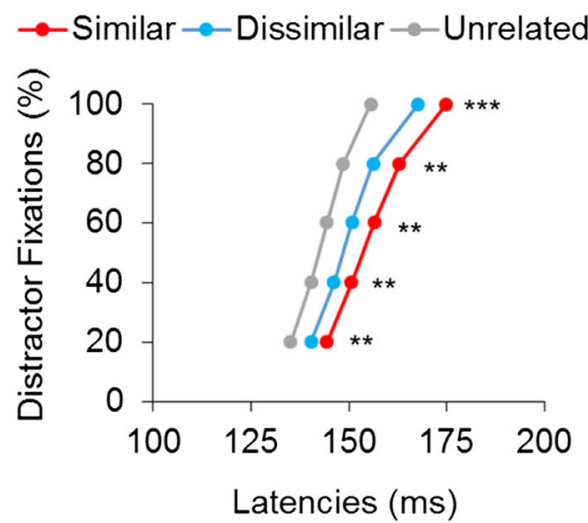

Fig. 3 A) Example of a trial in Experiment 2. The colour of the target was announced by a word cue (e.g., RED, GREEN). Observers had to make a fast and precise eye movement to the target and ignore the irrelevant onset, which was a red, green or blue square. B) Mean proportion of first eye movements to each of the three onset distractors (similar, dissimilar, unrelated) in Experiment 2, depicted separately for trials in which the target and/or onset distractor were repeated (Rep) vs. not repeated (NonRep). As shown by the asterisks, the target-similar onset was selected significantly more frequently than the targetdissimilar and unrelated distractor across all repetition conditions. C)

too, increased from the earliest bin to the last bin $(5 \mathrm{~ms}$ and $12 \mathrm{~ms}$, respectively). ${ }^{1}$

Again, interpretation of these results is complicated by the fact that the bins comprised different trial numbers: On average, the $20 \%$ fastest eye movements comprised 20.3 saccades to the similar distractor, which was a significantly higher number than

\footnotetext{
${ }^{1}$ In the cumulative latency distribution, the measurements in the different bins are not independent of each other, which can be problematic for statistical analysis and interpretation. However, computing the analysis in a noncumulative fashion (i.e., $20 \%$ fastest to $20 \%$ slowest saccades, in $20 \%$ steps) showed similar results, with saccades to the similar onset being significantly delayed compared to the dissimilar and unrelated distractor, across all latency bins (all $p \mathrm{~s}<0.05$ ), and the latency difference increasing from the earliest $20 \%$ saccades $(7 \mathrm{~ms})$ to the slowest $20 \%$ saccades (24 ms).
}

\section{D) Non-Cumulative Distribution}

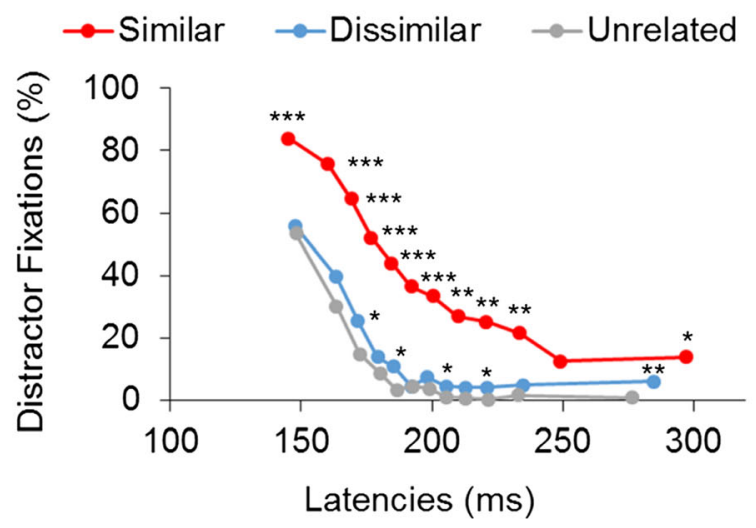

Mean latencies of fixating the onset distractors, depicted as a function of the saccadic latencies (20-100\% earliest saccades, in steps of $20 \%)$. As shown by the asterisks, saccades to the target-similar distractor were significantly delayed, compared with the target-dissimilar distractor. D) Mean proportion of first eye movements to the different onsets, as a function of the mean latencies of saccades to target or distractor (separated into 12 latency bins). Error bars depict the Standard Error of the Mean (SEM). $* p<0.05, * * p<0.01, * * * p<0.001$, as per two-tailed $t$ tests.

the 7.0 saccades to the dissimilar distractor, $t(11)=7.0, p<0.001$, and the 5.0 saccades to the unrelated distractor, $t(11)=8.2, p<$ 0.001 , and there were more saccades to the dissimilar than the unrelated distractor, $t(11)=2.7, p<0.021$.

Noncumulative Latency Distribution Analysis The noncumulative latency distribution comprised 12 latency bins to allow assessing saccades with latencies below $150 \mathrm{~ms}$ and test whether these were already modulated by top-down processes. As in Experiment 1, we first ordered all trials according to their saccadic latencies separately for each participant and distractor condition (from the shortest to the longest latencies; including eye movements to the distractor and the target). Figure $3 \mathrm{D}$ depicts the proportion of distractor fixations as a 
function of the target/distractor fixation latencies. A $3 \times 12$ ANOVA computed over the proportion of distractor fixations showed significant main effects for the distractor type (similar, dissimilar, unrelated), $F(2,22)=46.6, p<0.001, \eta^{2}=0.81$, the saccadic latency bin $(1-12), F(11,121)=91.8, p<0.001, \eta^{2}=$ 0.89 , and a significant interaction, $F(22,242)=6.0, p<0.001$, $\eta^{2}=0.35$. The interaction was due to the fact that the similar distractor was selected significantly more frequently than the dissimilar and unrelated distractor with early saccades (latency bins 1-10; mean difference: 17-50\%) and that this effect declined towards the later latency bins $(11,12$; mean difference: 8-13\%; Fig. 3D). Importantly, the earliest saccades (bin 1) showed significantly higher selection rates of the targetsimilar onset than the dissimilar onset, $t(13)=4.9, p<$ 0.001 , and the unrelated onset, $t(13)=5.1, p<0.001$, whereas the dissimilar and unrelated onsets did not differ, $t<1$.

The same $3 \times 12$ ANOVA computed over the latencies of target/distractor fixations showed a significant main effect of the latency bin, $F(11,143)=475.6, p<0.001, \eta^{2}=0.97$, and a significant interaction between distractor type and latency bin, $F(22,286)=9.1, p<0.001, \eta^{2}=0.41$. The latencies did not differ between distractors across the first 8 latency bins, whereas the presence of a similar distractor significantly delayed eye movements in bins 9-12, all $t \mathrm{~s}>2.1, p \mathrm{~s}<0.05$ compared with both the dissimilar and unrelated distractor. The (theoretically important) latencies in the $8 \%$ earliest saccades did not differ significantly between the onsets, all $t \mathrm{~s}<$ $1.5, p \mathrm{~s}<0.17$. This also was the case when only the distractor fixation latencies were compared (similar: $148 \mathrm{~ms}$; dissimilar: $148 \mathrm{~ms}$, unrelated: $147 \mathrm{~ms}$ ), all $t \mathrm{~s}<1$.

\section{Discussion}

Experiment 2 showed that attention can be top-down tuned to colour information provided by a word cue and that this strongly modulates capture by irrelevant onset distractors. Onsets are widely believed to affect the visual system at a very early stage of processing as they create a strong bottom-up signal (Yantis \& Jonides, 1984). Yet, Experiment 2 showed that top-down settings effectively modulate selection of onset distractors.

Top-down modulation of onset capture in Experiment 2 also cannot be attributed to automatic priming effects: As shown in Fig. 3B, the similar distractor was selected significantly more frequently than the dissimilar distractor even when it did not match the previous target colour. In fact, capture by the similar distractor was not modulated at all by the previous target colour, which rules out priming effects and indicates that capture by the similar distractor was due to top-down tuning to the target colour.

The results of the cumulative latency distribution analysis replicated the findings of Mulckhuyse et al. (2008), in that the
$20 \%$ earliest saccades to the dissimilar distractors had slightly shorter latencies than saccades to the similar distractor. Still, both latency distribution analyses (cumulative, noncumulative) showed a higher proportion of saccades to the similar onset than the dissimilar or unrelated onsets, including at an early stage. The noncumulative distribution analysis moreover showed that similar onsets were more frequently selected within the $8 \%$ fastest eye movements (with latencies $<150 \mathrm{~ms}$ ) and without any differences in saccadic latencies. These findings are inconsistent with the dual stage account (Mulckhuyse et al., 2008) and show that onset capture is modulated by top-down processes at an early stage, in line with a feedforward account (Ansorge et al., 2010; Folk et al., 1992).

\section{General Discussion}

The present study shows that top-down expectations affect visual selection at an early stage of visual processing and thus provides strong evidence for a feedforward account of topdown control. This contrasts with earlier studies that found that the earliest saccades were not modulated by top-down knowledge, in support of a pure feedback account of topdown control. Perhaps the strongest evidence for the feedback account was provided by Born et al. (2011), who found no preferential selection of target-similar onset distractors when the mean saccadic latencies were below $150 \mathrm{~ms}$. However, Experiment 1 showed that the red premasks used in the study of Born et al. (2011) produce a colour after-effect that can render a differently coloured onset distractor similar to the (perceived) colour of the target. Hence, the critical comparisons in the study of Born et al. (2011) may have involved two target-similar onset distractors - one that was similar to the subjective appearance of the target (due to a colour-after-effect), and one that had the target colour (grey). With this, previous studies cannot clearly distinguish between topdown vs. bottom-up processes and thus, cannot provide compelling evidence for the absence of top-down control at an early stage of visual search.

Other studies established that a target-similar onset is not selected earlier than target-dissimilar onsets, which seemed to contradict the view that top-down tuning to the target colour increases the saliency of target-similar items (Mulckhuyse et al., 2008). However, these studies overlooked that a topdown bias could also be reflected in a higher probability of selecting target-similar items rather than the speed of saccades (i.e., saccadic latency).

The present study provided clear evidence that early saccades are modulated by both top-down knowledge and bottom-up saliency. Both Experiments 1 and 2 showed that early saccades were more likely to be directed to the onset distractor than to the target (including when the onset distractor had a dissimilar colour; Figs. 2C, 3D). These results 
are consistent with the view that suddenly appearing onsets can attract the gaze due to being visually salient events (Theeuwes et al., 2000; Yantis \& Jonides, 1984). However, the results also showed a strong effect of top-down tuning, as the target-similar onset distractor was far more likely to attract the gaze than the target-dissimilar onset. Moreover, the results also indicated that top-down effects were particularly strong for eye movements that were launched early, within $\sim 130$ to $\sim 200 \mathrm{~ms}$ (Fig. 3D; bins 1-5). These results suggest that topdown knowledge affects visual selection at an early stage and before the appearance of any stimuli (i.e., in a feedforward manner).

Importantly, a top-down bias for a particular colour only changed the probability of reflexively selecting targetmatching onset distractors, without speeding up respective eye movements (see also Mulckhuyse et al., 2008). Still, this should not be taken to mean that the earliest saccades are driven by bottom-up processes and that top-down processes modulate selection only at a later stage. In this instance, the earliest eye movements should have been equally likely to select target-similar and dissimilar onset distractors. Yet, there was no point in time in which saccades to similar and dissimilar distractors were equally likely, indicating that selection was consistently modulated by top-down information, including at an early stage.

The finding that the earliest saccades to target-similar onsets are typically delayed (Mulckhuyse et al., 2008) also seems consistent with feedforward models of visual selection. According to the Guided Search model (Wolfe, 1994), for instance, visual selection is driven by activation signals on an integrated saliency map (Wolfe, 1994), which is jointly determined by bottom-up processes and top-down knowledge. If we assume that it takes time for these activation signals to build up, it follows automatically that saccades to target-dissimilar distractors will be launched earlier. Executing an eye movement at an early point in time requires a lower saccade threshold that cannot distinguish between two salient items. To discriminate between target-similar and dissimilar salient onset items, a higher saccade threshold is necessary, which can only be surpassed by a target-similar item but not a target-dissimilar item. Setting the threshold to such a higher value will however delay the eye movement (as it takes time for the activation signals to build up on the saliency map). Hence, pure feedforward models, such as Guided Search, can account for the typical findings that eye movements to targetsimilar onsets occur on average $15-53 \mathrm{~ms}$ later than eye movements to target-dissimilar onsets, and that even the $20 \%$ earliest saccades to target-similar items are slightly delayed (Ludwig \& Gilchrist, 2002; Mulckhuyse et al., 2008).

In the present study, we extracted the earliest eye movements and analysed the proportion of eye movements to either onset distractor. The results clearly showed that the $8 \%$ earliest saccades were already biased to the target-similar onset distractor. These results indicate that top-down knowledge about the target colour modulates the activation signals on the saliency map at an early stage, by increasing the saliency of target-coloured items within the feedforward sweep of processing. Deviating from previous studies, these top-down effects also cannot be attributed to priming, as the target colour randomly varied and observers were informed about the upcoming target colour by a word cue (Fig. 3A). Thus, the results provide strong evidence that the earliest saccades are modulated by top-down knowledge.

With this, the present results strongly support a feedforward account of top-down tuning, in which bottomup and top-down processes together determine the attentiondriving capacity of a stimulus and influence visual selection at an early stage of visual processing (Ludwig \& Gilchrist, 2002; Wolfe, 1994).

This conclusion is in line with previous neurophysiological studies that have shown that the expectation of a specific target colour already modulates the activity of colour-sensitive neurons in the monkey (Motter, 1994) and in humans (Beck \& Kastner, 2009; Kastner et al., 1999) even in the absence of any stimuli, so that target-matching stimuli can elicit a stronger neural response once the stimuli are presented (during the feedforward sweep). The challenge for future research is to describe how exactly top-down expectations modulate feature-specific neurons in advance of sensory inputs, to cause the observed top-down effects on visual selection.

Acknowledgments This research was supported by an Australian Research Council (ARC) Future Fellowship (FT130101282), and a University of Queensland Foundation Research Excellence Award awarded to Stefanie I. Becker. Correspondence to Stefanie I. Becker, School of Psychology, The University of Queensland, St Lucia QLD 4072, Australia. Email: s.becker@psy.uq.edu.au.

\section{References}

Ansorge, U., Horstmann, G., \& Scharlau, I. (2010). Top-down contingent attentional capture during feed-forward visual processing. Acta Psychologica, 135, 123-126.

Bacon, W. F., \& Egeth, H. E. (1994). Overriding stimulus-driven attentional capture. Perception \& Psychophysics, 55, 485-496.

Beck, D. M., \& Kastner, S. (2009). Top-down and bottom-up mechanisms in biasing competition in the human brain. Vision Research, 49, 1154-1165.

Becker, S. I. (2010). Oculomotor capture by irrelevant colour singletons depends on intertrial priming. Vision Research, 50, 2116-2126.

Becker, S. I. (2008). The stage of priming: Are intertrial repetition effects attentional or decisional? Vision Research, 48, 664-684.

Becker, S. I. (2007). Irrelevant singletons in pop-out search: Attentional capture or filtering costs? Journal of Experimental Psychology: Human Perception and Performance, 33, 764-787.

Becker, S. I., Ansorge, U., \& Horstmann, G. (2009). Can intertrial priming account for the similarity effect in visual search? Vision Research, 49, 1738-1756. 
Becker, S. I., \& Lewis, A. J. (2015). Oculomotor capture by irrelevant onsets with and without color contrast. Annals of the New York Academy of Sciences, 1339, 60-71.

Born, S., Kerzel, D., \& Theeuwes, J. (2011). Evidence for a dissociation between the control of oculomotor capture and disengagement. Experimental Brain Research, 208, 621-631.

Folk, CL \& Remington, RW (1998). Selectivity in distraction by irrelevant feature singletons: Evidence for two forms of attentional capture. Journal of Experimental Psychology: Human Perception and Performance, 24(3), 847-858.

Folk, C. L., \& Anderson, B. A. (2010). Target-uncertainty effects in attentional capture: Color-singleton set or multiple attentional control settings? Psychonomic Bulletin \& Review, 17, 421-426.

Folk, C. L., Remington, R. W., \& Johnston, J. C. (1992). Involuntary covert orienting is contingent on attentional control settings. Journal of Experimental Psychology: Human Perception and Performance, 18, 1030-1044.

Geyer, T., Mueller, H. J., \& Krummenacher, J. (2008). Expectancies modulate attentional capture by salient color singletons. Vision Research, 48, 1315-1326.

Harris, A. M., Becker, S. I., \& Remington, R. W. (2015). Capture by colour: Evidence for dimension-specific singleton capture. Attention, Perception, \& Psychophysics, 77, 2305-2321.

Kastner, S., Pinsk, M. A., De Weerd, P., Desimone, R., \& Ungerleider, L. G. (1999). Increased activity in human visual cortex during directed attention in the absence of visual stimulation. Neuron, 22, 751-761.

Lavie, N., Hirst, A., de Fockert, J. W., \& Viding, E. (2004). Load theory of selective attention and cognitive control. Journal of Experimental Psychology: General, 133, 339-354.

Ludwig, C. J. H., \& Gilchrist, I. D. (2002). Stimulus-driven and goaldriven control over visual selection. Journal of Experimental Psychology: Human Perception and Performance, 28, 902-912.

Maljkovic, V., \& Nakayama, K. (1994). Priming of pop-out: I. Role of features. Memory \& Cognition, 22, 657-672.

Moher, J., Abrams, J., Egeth, H. E., Yantis, S., \& Stuphorn, V. (2011). Trial-by-trial adjustments of top-down set modulate oculomotor capture. Psychonomic Bulletin \& Review, 18, 897-903.
Motter, B. C. (1994). Neural correlates of attentive selection for color and luminance in extrastriate area V4. The Journal of Neuroscience, 14, $2178-2189$.

Mulckhuyse, M., Van Zoest, W., \& Theeuwes, J. (2008). Capture of the eyes by relevant and irrelevant onsets. Experimental Brain Research, 186, 225-235.

Sayim, B., Grubert, A., Herzog, M. H., \& Krummenacher, J. (2010). Display probability modulates attentional capture by onset distractors. Journal of Vision, 10, 1-8.

Theeuwes, J. (2013). Feature-based attention: It is all bottom-up priming. Philosophical Transactions of the Royal Society of London B, 368, 20130055.

Theeuwes, J. (2004). Top-down search strategies cannot override attentional capture. Psychonomic Bulletin \& Review, 11, 65-70.

Theeuwes, J. (1994). Stimulus-driven capture and attentional set: Selective search for color and visual abrupt onsets. Journal of Experimental Psychology: Human Perception and Performance, 20, 799-806.

Theeuwes, J. (1992). Perceptual selectivity for color and form. Perception \& Psychophysics, 51, 599-606.

Theeuwes, J., Atchley, P., \& Kramer, A. F. (2000). On the time course of top-down and bottom-up control of visual attention (p. 105-125). In S. Monsell \& J. Driver (Eds.), Attention \& performance (Vol 18). Cambridge: MIT Press.

Theeuwes, J., \& Lucassen, M. P. (1993). An adaptation-induced pop-out in visual search. Vision Research, 33, 2353-2357.

Van Zoest, W., Donk, M., \& Theeuwes, J. (2004). The role of stimulusdriven and goal-driven control in saccadic visual selection. Journal of Experimental Psychology: Human Perception and Performance, 30, 746-759.

Wolfe, J. M. (1994). Guided Search 2.0: A revised model of visual search. Psychonomic Bulletin \& Review, 1, 202-238.

Wu, S.-C., \& Remington, R. W. (2003). Characteristics of covert and overt visual orienting: Evidence from attentional and oculomotor capture. Journal of Experimental Psychology: Human Perception and Performance, 29, 1050-1067.

Yantis, S., \& Jonides, J. (1984). Abrupt visual onsets and selective attention: Evidence from visual search. Journal of Experimental Psychology: Human Perception and Performance, 10, 601-621. 